\title{
RESOLUTION OF BOUNDARY PROBLEMS BY THE USE OF A GENERALIZED CONVOLUTION
}

\author{
R. C. F. BARTELS AND R. V. CHURCHILL
}

1. The Laplace transformation of the convolution. The generalized convolution $F^{*}(t)$ of $F\left(t, t^{\prime}\right)$ is defined as follows:

$$
F^{*}(t)=\int_{0}^{t} F\left(t-t^{\prime}, t^{\prime}\right) d t^{\prime}
$$

In case $F\left(t, t^{\prime}\right)=F_{1}(t) F_{2}\left(t^{\prime}\right)$, the function $F^{*}(t)$ is the ordinary convolution $F_{1} * F_{2}$, or Faltung, ${ }^{1}$ of the two functions $F_{1}$ and $F_{2}$.

Let $L\left\{F^{*}(t)\right\}$ denote the Laplace transform of $F^{*}$ with respect to $t$,

$$
L\left\{F^{*}(t)\right\}=\int_{0}^{\infty} e^{-s t} F^{*}(t) d t
$$

and let $\bar{f}(s)$ denote the iterated transform of $F\left(t, t^{\prime}\right)$,

$$
\bar{f}(s)=\int_{0}^{\infty} e^{-s t^{\prime}} d t^{\prime} \int_{0}^{\infty} e^{-s t} F\left(t, t^{\prime}\right) d t .
$$

It will be seen that

$$
L\left\{F^{*}(t)\right\}=\bar{f}(s),
$$

which, in terms of the inverse Laplace transformation, implies that

$$
L^{-1}\{\bar{f}(s)\}=F^{*}(t) .
$$

THEOREM. Let $F\left(t, t^{\prime}\right)$ be an integrable function of $t$ and $t^{\prime}$ in every finite rectangle $0 \leqq t \leqq T, 0 \leqq t^{\prime} \leqq T^{\prime}$ and, for some real $\alpha$, let $e^{-\alpha\left(t+t^{\prime}\right)}\left|F\left(t, t^{\prime}\right)\right|$ be bounded for all $t$ and $t^{\prime}\left(t \geqq 0, t^{\prime} \geqq 0\right)$. Then if $R(s)>\alpha$, the integral $L\left\{F^{*}(t)\right\}$ is absolutely convergent and satisfies the equation (2).

Under the conditions stated, the iterated integral in (1) exists if $R(s)>\alpha$ and is equal to the absolutely convergent double integral

$$
\iint e^{-s\left(t+t^{\prime}\right)} F\left(t, t^{\prime}\right) d\left(t, t^{\prime}\right)
$$

over the quadrant $t \geqq 0, t^{\prime} \geqq 0$. However, the latter is equal to

Presented to the Society, April 12, 1940, under the title An extension of Duhamel's theorem; received by the editors March 28, 1941.

${ }^{1}$ G. Doetsch, Theorie und Andwendung der Laplace-Transformation, Berlin, 1937, p. $155 \mathrm{ff}$. 


$$
\lim _{k \rightarrow \infty} \iint_{T_{k}} e^{-s\left(t+t^{\prime}\right)} F\left(t, t^{\prime}\right) d\left(t, t^{\prime}\right),
$$

where $T_{k}$ is the triangle bounded by the lines $t^{\prime}=0, t=0$ and $t+t^{\prime}=k$. After the substitutions $\tau=t+t^{\prime}, \tau^{\prime}=t^{\prime}$ have been made, the integral in (3) is transformed into the integral over the triangle bounded by the lines $\tau^{\prime}=0, \tau=\tau^{\prime}$, and $\tau=k$ in the $\tau \tau^{\prime}$-plane. Hence (3) can be written

$$
\lim _{k \rightarrow \infty} \int_{0}^{k} e^{-s \tau} d \tau \int_{0}^{\tau} F\left(\tau-\tau^{\prime}, \tau^{\prime}\right) d \tau^{\prime},
$$

which is $L\left\{F^{*}(t)\right\}$. Therefore the equality in (2) holds.

The convergence of the integral $L\left\{\left|F^{*}(t)\right|\right\}$, that is, the absolute convergence of the Laplace integral in (2), follows immediately from the absolute convergence of the limit in (3).

2. The Duhamel integral formula. Let $\Lambda$ and $\lambda$ denote the differential operators defined as follows:

$$
\begin{aligned}
& \Lambda\{U\}=C_{0} U+\sum_{i=1}^{3} C_{i} \frac{\partial}{\partial x_{i}}\left(K_{i} \frac{\partial U}{\partial x_{i}}\right), \\
& \lambda\{U\}=c_{0} U+\sum_{i=1}^{3} c_{i} \frac{\partial U}{\partial x_{i}}
\end{aligned}
$$

where the coefficients of $U$ and its derivatives are functions of $x_{1}, x_{2}$, and $x_{3}$ only. Then, if $P$ denotes an arbitrary interior point $\left(x_{1}, x_{2}, x_{3}\right)$ of a region $R$, and $Q$ any point on the boundary $S$ of $R$, a general boundary value problem for the temperature $U(P, t)$ in the region $R$ can be written

$$
\begin{array}{rlrl}
\frac{\partial}{\partial t} U(P, t) & =\Lambda\{U\}+F(P, t), & & t>0, \\
\lambda\{U(Q, t)\} & =G(Q, t), & & t>0, \\
U(P, 0) & =H(P), &
\end{array}
$$

where $F, G$, and $H$ are prescribed functions. In the second of these equations it is understood that $\lambda\{U(Q, t)\}$ represents the limit of $\lambda\{U(P, t)\}$ as $P$ approaches the point $Q$ on $S$ in a prescribed manner. Similarly, in the last equation $U(P, 0)$ is written for $U(P,+0)$.

Applying the Laplace transformation with respect to $t$ to the first two equations in problem (A) gives, in view of the last condition, 


$$
\begin{aligned}
s u(P, s)-H(P) & =\Lambda\{u(P, s)\}+f(P, s), \\
\lambda\{u(Q, s)\} & =g(Q, s),
\end{aligned}
$$

where $u, f$, and $g$ are the transforms of $U, F$, and $G$, respectively.

Let the temperature function $V\left(P, t, t^{\prime}\right)$, depending on the fixed parameter $t^{\prime}$, be the solution of problem (A) when the source function $F$ and the surface-temperature function $G$ have at each point of $R$ and $S$, respectively, the constant values $F\left(P, t^{\prime}\right)$ and $G\left(Q, t^{\prime}\right)$. Then

$$
\begin{aligned}
\frac{\partial}{\partial t} V\left(P, t, t^{\prime}\right) & =\Lambda\left\{V\left(P, t, t^{\prime}\right)\right\}+F\left(P, t^{\prime}\right), \\
\lambda\left\{V\left(Q, t, t^{\prime}\right)\right\} & =G\left(Q, t^{\prime}\right), \\
V\left(P, 0, t^{\prime}\right) & =H(P) .
\end{aligned}
$$

Also let $v\left(P, s, t^{\prime}\right)$ represent the Laplace transformation of $V\left(P, t, t^{\prime}\right)$ with respect to $t$, and $\bar{v}(P, s)$ the iterated transform of $V\left(P, t, t^{\prime}\right)$ with respect to $t$ and $t^{\prime}$. Then applying the transformation with respect to $t$, it follows from (B) that

$$
\begin{aligned}
s v\left(P, s, t^{\prime}\right)-H(P) & =\Lambda\left\{v\left(P, s, t^{\prime}\right)\right\}+\frac{1}{s} F\left(P, t^{\prime}\right), \\
\lambda\left\{v\left(Q, s, t^{\prime}\right)\right\} & =\frac{1}{s} G\left(Q, t^{\prime}\right),
\end{aligned}
$$

and consequently, on applying the transformation with respect to $t^{\prime}$ to these equations, that

$$
s \bar{v}(P, s)-\frac{1}{s} H(P)=\Lambda\{\bar{v}(P, s)\}+\frac{1}{s} f(P, s),
$$

$$
\lambda\{\bar{v}(Q, s)\}=\frac{1}{s} g(Q, s) .
$$

Upon multiplying each member of the equations in $\left(\mathrm{B}^{\prime}\right)$ by $s$, it is at once evident that the problems $\left(\mathrm{A}^{\prime}\right)$ and $\left(\mathrm{B}^{\prime}\right)$ are equivalent and that the function $s \bar{v}(P, s)$ is a solution of $\left(\mathrm{A}^{\prime}\right)$. Thus, assuming that the solution of $\left(\mathrm{A}^{\prime}\right)$ is unique, it follows that

$$
u(P, s)=s \bar{v}(P, s) .
$$

According to equation (2),

$$
\bar{v}(P, s)=L\left\{V^{*}(P, t)\right\}=L\left\{\int_{0}^{t} V(P, t-\tau, \tau) d \tau\right\} .
$$


Moreover, since $V^{*}(P, 0)=0$,

$$
L\left\{\frac{\partial V^{*}}{\partial t}\right\}=s \bar{v}(P, s) .
$$

Hence it follows from equation (4), on performing the inverse transformation, that

$$
U(P, t)=\frac{\partial}{\partial t} \int_{0}^{t} V(P, t-\tau, \tau) d \tau .
$$

The solution of the problem (A) with variable source and surface conditions is therefore given by formula (5) in terms of the solution of the problem (B) with constant source and surface conditions.

Sufficient conditions can, of course, be given in order to justify all of the steps in the formal derivation of (5). However, more relaxed conditions can be obtained in particular cases by verifying directly that the function given by (5) is a solution of problem (A). When the latter procedure is applied to the above general case, it is seen that the foregoing result in italics is true, provided that $V, \partial V / \partial x, \partial^{2} V / \partial x_{i}^{2}$, and their derivatives with respect to $t$ are continuous functions of $P, t$, and $t^{\prime}$ interior to $R$ when $t \geqq 0$ and $t^{\prime} \geqq 0$, and that the function $(\partial / \partial t) \int_{0}^{t} \lambda\{V(P, t-\tau, \tau)\} d \tau$ is continuous with respect to $P$ at the points $Q$ when $t>0$.

The relation (5) is known in the theory of heat conduction as Duhamel's integral formula. ${ }^{2}$ It has been shown above that this formula applies to a very general temperature problem. It is clear that the procedure can be applied in case of discontinuous media, and to other boundary value problems as well. It is, of course, applicable to problems with partial differential equations of higher order than the second.

3. Resolution of temperature problems. It will now be shown that the general problem (A) can be further resolved into still simpler problems which are of two basic types. It should first be observed that the solution of problem (B) can be written

$$
V\left(P, t, t^{\prime}\right)=V_{1}\left(P, t^{\prime}\right)+V_{2}\left(P, t, t^{\prime}\right)+W\left(P, t, t^{\prime}\right),
$$

where $V_{1}, V_{2}$, and $W$ are solutions of the problems:

$$
\text { (a) } \quad \Lambda\left\{V_{1}\left(P, t^{\prime}\right)\right\}=0, \quad \lambda\left\{V_{1}\left(Q, t^{\prime}\right)\right\}=G\left(Q, t^{\prime}\right) \text {; }
$$

${ }^{2} \mathrm{~J}$. M. C. Duhamel, Mémoir sur la mêthode générale relative au mouvement de le chaleur dans les corps solides plongers dans des milieux dont la température varie avec le temps, Journal de l'École Polytechnique, vol. 14 (1830), pp. 20-77; also see H. S. Carslaw, Conduction of Heat, 1921, pp. 16-19. 
(b)

$$
\begin{gathered}
\frac{\partial}{\partial t} V_{2}\left(P, t, t^{\prime}\right)=\Lambda\left\{V_{2}\left(P, t, t^{\prime}\right)\right\}, \\
\lambda\left\{V_{2}\left(Q, t, t^{\prime}\right)\right\}=0, \quad V_{2}\left(P, 0, t^{\prime}\right)=H(P)-V_{1}\left(P, t^{\prime}\right) ;
\end{gathered}
$$

and

$$
\begin{gathered}
\frac{\partial}{\partial t} W\left(P, t, t^{\prime}\right)=\Lambda\left\{W\left(P, t, t^{\prime}\right)\right\}+F\left(P, t^{\prime}\right), \\
\lambda\left\{W\left(Q, t, t^{\prime}\right)\right\}=0, \quad W\left(P, 0, t^{\prime}\right)=0 .
\end{gathered}
$$

In terms of the transform $w\left(P, s, t^{\prime}\right)$ of $W\left(P, t, t^{\prime}\right)$, the last of the foregoing problems becomes

$$
s w\left(P, s, t^{\prime}\right)-\frac{1}{s} F\left(P, t^{\prime}\right)=\Lambda\left\{w\left(P, s, t^{\prime}\right)\right\}, \quad \lambda\left\{w\left(Q, s, t^{\prime}\right)\right\}=0 .
$$

On multiplying each member of these two equations by $s$, it is immediately evident that the function $\operatorname{sw}\left(P, s, t^{\prime}\right)$ is a solution of the transform of the following problem:

$$
\begin{gathered}
\frac{\partial}{\partial t} V_{3}\left(P, t, t^{\prime}\right)=\Lambda\left\{V_{3}\left(P, t, t^{\prime}\right)\right\}, \\
\lambda\left\{V_{3}\left(Q, t, t^{\prime}\right)\right\}=0, \quad V_{3}\left(P, 0, t^{\prime}\right)=F\left(P, t^{\prime}\right) .
\end{gathered}
$$

Therefore, if $v_{3}\left(P, s, t^{\prime}\right)$ denotes the transform of $V_{3}\left(P, t, t^{\prime}\right)$, then $w=v_{3} / s$ and, consequently,

$$
W\left(P, t, t^{\prime}\right)=\int_{0}^{t} V_{3}\left(P, \tau, t^{\prime}\right) d \tau .
$$

According to equations (5), (6), and (7), the solution of problem (A) is given in terms of the solutions $V_{1}, V_{2}$, and $V_{3}$ of the problems (a), (b), and (c), respectively, as follows:

which can be written

$$
\begin{aligned}
U(P, t)= & \frac{\partial}{\partial t} \int_{0}^{t}\left[V_{1}(P, \tau)+V_{2}(P, t-\tau, \tau)\right. \\
& \left.+\int_{0}^{t-\tau} V_{3}\left(P, \tau^{\prime}, \tau\right) d \tau^{\prime}\right] d \tau,
\end{aligned}
$$

$$
\begin{aligned}
U(P, t)= & V_{1}(P, t)+\frac{\partial}{\partial t} \int_{0}^{t} V_{2}(P, t-\tau, \tau) d \tau \\
& +\int_{0}^{t} V_{3}(P, t-\tau, \tau) d \tau .
\end{aligned}
$$


Problems (b) and (c) are variable state problems of the same type in which the initial condition alone is nonhomogeneous. On the other hand, (a) is a steady state problem. The general temperature problem (A) is thus resolved by formula (8) into the solution of problems of two basic types, one of steady state, the other, of variable state.

4. Resolution of vibration problems. A general form of the problem of displacements in elastic media with variable boundary conditions, including forced vibrations of membranes, shafts, and strings, can be written

$$
\begin{gathered}
\frac{\partial^{2}}{\partial t^{2}} \Phi(P, t)+b \frac{\partial}{\partial t} \Phi(P, t)=\Lambda\{\Phi(P, t)\}+F(P, t), \\
\lambda\{\Phi(Q, t)\}=G(Q, t), \\
\Phi(P, 0)=H(P), \quad \frac{\partial}{\partial t} \Phi(P, 0)=I(P) .
\end{gathered}
$$

As before, $P$ denotes a point in a region $R$, and $Q$ a point on the boundary of $R ; F, G, H, I$, and $b$ are prescribed functions, $b$ being a function of $P$ alone. The resolution of this problem can, as in the case of the foregoing temperature problem, be easily obtained by formal application of the Laplace transformation. The problem corresponding to (C) in $\phi(P, s)$, the transform of $\Phi(P, t)$, is

$$
\begin{gathered}
\left(s^{2}+b s\right) \phi(P, s)-(s+b) H(P)-I(P)=\Lambda\{\phi(P, s)\}+f(P, s), \\
\lambda\{\phi(Q, s)\}=g(Q, s) .
\end{gathered}
$$

Let the function $\Theta\left(P, t, t^{\prime}\right)$, depending upon the fixed parameter $t^{\prime}$, be the solution of problem (C) when $F$ and $G$ have at each point the constant values $F\left(P, t^{\prime}\right)$ and $G\left(Q, t^{\prime}\right)$, respectively, that is,

$$
\begin{aligned}
& \frac{\partial^{2}}{\partial t^{2}} \Theta\left(P, t, t^{\prime}\right)+b \frac{\partial}{\partial t} \Theta\left(P, t, t^{\prime}\right)=\Lambda\left\{\Theta\left(P, t, t^{\prime}\right)\right\}+F\left(P, t^{\prime}\right), \\
& \lambda\left\{\Theta\left(Q, t, t^{\prime}\right)\right\}=G\left(Q, t^{\prime}\right), \\
& \Theta\left(P, 0, t^{\prime}\right)=H(P), \quad \frac{\partial}{\partial t} \Theta\left(P, 0, t^{\prime}\right)=I(P) .
\end{aligned}
$$

If $\bar{\theta}(P, s)$ is the iterated transform of $\Theta\left(P, t, t^{\prime}\right)$, it follows from (D) that $s \bar{\theta}$ satisfies the equations $\left(\mathrm{C}^{\prime}\right)$. Therefore, $\phi(P, s)=s \bar{\theta}(P, s)$ and, according to (2), we have the formula

$$
\Phi(P, t)=\frac{\partial}{\partial t} \int_{0}^{t} \Theta(P, t-\tau, \tau) d \tau
$$


Repeating the procedure of the preceding section, it is seen that

$$
\Theta\left(P, t, t^{\prime}\right)=\Theta_{1}\left(P, t^{\prime}\right)+\Theta_{2}\left(P, t, t^{\prime}\right)+\int_{0}^{t} \Theta_{3}\left(P, \tau, t^{\prime}\right) d \tau
$$

where $\Theta_{1}, \Theta_{2}$, and $\Theta_{3}$ are the solutions of the following problems, respectively,

$$
\begin{gathered}
\left.\Lambda_{\{} \Theta_{1}\left(P, t^{\prime}\right)\right\}=0, \quad \lambda\left\{\Theta_{1}\left(Q, t^{\prime}\right)\right\}=G\left(Q, t^{\prime}\right) \\
\frac{\partial^{2}}{\partial t^{2}} \Theta_{2}\left(P, t, t^{\prime}\right)+b \frac{\partial}{\partial t} \Theta_{2}\left(P, t, t^{\prime}\right)=\Lambda\left\{\Theta_{2}\left(P, t, t^{\prime}\right)\right\},
\end{gathered}
$$

(e)

$$
\begin{gathered}
\lambda\left\{\Theta_{2}\left(Q, t, t^{\prime}\right)\right\}=0, \quad \Theta_{2}\left(P, 0, t^{\prime}\right)=H(P)-\Theta_{1}\left(P, t^{\prime}\right), \\
\frac{\partial}{\partial t} \Theta_{2}\left(P, 0, t^{\prime}\right)=I(P) ;
\end{gathered}
$$

$$
\frac{\partial^{2}}{\partial t^{2}} \Theta_{3}\left(P, t, t^{\prime}\right)+b \frac{\partial}{\partial t} \Theta_{3}\left(P, t, t^{\prime}\right)=\Lambda\left\{\Theta_{3}\left(P, t, t^{\prime}\right)\right\},
$$

$$
\begin{gathered}
\lambda\left\{\Theta_{3}\left(Q, t, t^{\prime}\right)\right\}=0, \quad \Theta_{3}\left(P, 0, t^{\prime}\right)=0, \\
\frac{\partial}{\partial t} \Theta_{3}\left(P, 0, t^{\prime}\right)=F\left(P, t^{\prime}\right) .
\end{gathered}
$$

Therefore, in view of (9),

$$
\begin{aligned}
\Phi(P, t)= & \Theta_{1}(P, t)+\frac{\partial}{\partial t} \int_{0}^{t} \Theta_{2}(P, t-\tau, \tau) d \tau \\
& +\int_{0}^{t} \Theta_{3}(P, t-\tau, \tau) d \tau .
\end{aligned}
$$

Thus the solution of the general problem (C) is resolved into the solution of the two basic types (d), and (e). Problem (f) is a special case of (e). The latter can, of course, be still further resolved into two simpler problems, one being of the same form as (f) with the second initial condition nonhomogeneous, and the other having the nonhomogeneity occurring in the first initial condition.

The University of Michigan 